\title{
Half and half nail, is it a marker of severe COVID-19 infection?
}

SORAYA AOUALI ${ }^{1}$, Saida Sefraoui ${ }^{1}$, nada zizi $^{1}$, and Siham Dikhaye ${ }^{1}$

${ }^{1}$ Mohammed VI University Hospital at Oujda

August 25, 2021

\begin{abstract}
Half and half nail or Lindsay's nail is a rare clinical finding that can be seen in many chronic diseases, such as chronic renal failure. We report 2 cases of half and half nail aspect during severe Covid-19 infection suggesting that it can be considered as a severity marker.
\end{abstract}

Half and half nail, is it a marker of severe COVID-19 infection?

Soraya Aouali ${ }^{1}$, Saida Sefraoui ${ }^{1}$, Nada Zizi ${ }^{1,2}$, Siham Dikhaye ${ }^{1 ; 2}$

(1) Department of Dermatology, Mohammed the VI ${ }^{\text {th }}$ University Hospital of Oujda, Morocco

(2) Laboratory of Epidemiology, Clinical Research and Public Health, Faculty of Medicine and Pharmacy, Mohamed the first university of Oujda, Oujda, Morocco

Authors contribution:

Soraya Aouali:

- Correspending author

- Conception

- Writing manuscript

Saida Sefraoui :

Investigation

Nada Zizi :

Work supervision

Siham Dikhaye :

Work supervision

Correction of the manuscript

Abstract :

Half and half nail or Lindsay's nail is a rare clinical finding that can be seen in many chronic diseases, such as chronic renal failure. We report 2 cases of half and half nail aspect during severe Covid-19 infection suggesting that it can be considered as a severity marker.

Key clinical message : 
Half and half nail aspect or Lindsay nails is a rare clinical sign that can be seen in many chronic diseases, such as chronic renal failure. We report the first 2 cases of Lindsay nails occurring with severe COVID-19 infection.

\section{Introduction :}

Cutaneous manifestations related to COVID-19 have been widely reported since the coronavirus struck. Yet little is known about nail changes.

We report the cases of 2 patients that showed an ungual symptom, previously unreported, during and following COVID-19 infection.

Case $N^{\circ} 1$ :

An 82 year-old male, diabetic on insulin therapy, had been admitted to COVID-19 department. He was diagnosed with a severe COVID -19 pneumonia confirmed with a positive polymerase chain reaction (PCR) and chest tomography that showed $75 \%$ impairment scored CORADS 6 , with progressive deterioration of respiratory function motivating his transfer to intensive care 5 days after his admission. Laboratory findings showed lymphopenia and elevation of inflammation markers, kidney function remained normal during his hospitalization.

Two days prior to respiratory symptoms, the patient reported the appearance of finger nails changes. Physical examination found color anomaly of all finger-nails, with white proximal portion of the nail and red distal half in favour of Lindsay nails or half and half nail aspect. Dermoscopy gave a clear vision to the sharp line of demarcation between the 2 portions, in addition to some mega-capillaries.

The patient died in intensive care unit due to respiratory distress.

Case $N^{\circ} 2$ :

A 71 year-old women, diabetic on oral treatment, had been admitted to COVID-19 department for a severe infection; scored CORADS 6 with a positive PCR, 50 to $75 \%$ impairment in chest tomography and elevation of inflammation markers. One month later, she had been referred to our dermatology department for a pustular rash with facial edema. That was diagnosed as Acute Generalised Exanthematous Pustulosis to aspirin. Furthermore, physical examination showed half and half aspect in all finger nails evolving since the infectious episode.

Discussion :

Half and half nail also known as Lindsay's nail was first described by Lindsay in 1967 (1). Clinically, it shows as a nail with white proximal portion and reddish brown or pink distal portion, with a sharp demarcation line between the two portions, which usually remains parallel to the distal free margin of the nail. It is a rare clinical finding, but it can be frequently seen in chronic renal failure. It can also appear in yellow nails syndrome, Crohn's disease, Kawasaki's disease, Behcet's disease, cirrhosis, hyperthyroidism, zinc deficiency, citrullinemia, pellagra and HIV infection (2).

During the COVID-19 pandemic, several cutaneous manifestations have been reported. Recently ; nail changes started getting attention, beginning with transverse leukonychia (Mees lines) (3); to red half-moon nail sign (4) and Beau lines (5). These nail alterations can be explained by keratinization anomalies of nail tablet associated to vascularization alterations of nail bed. A transient matrix injury caused by COVID-19 infection is suggested.

\section{Conclusion :}

Our cases report the first description of acute appearence of half and half nail aspect during COVID-19 severe infection in 2 patients without history of kidney failure or any other condition previously described.

Conflict of interest: None. 
This case report has been published as an e-poster in the EADV spring symposium.

References :

1 : Lindsay PG. The Half-and-Half nail. Arch Intern Med. 1967;119:583-7.

2 : Iorizzo M, Daniel CR, Tosti A. Half and half nails: A past and present snapshot. Cutis. 2011;88:138-9.

3: Diego Fernandez-Nieto, Juan Jimenez-Cauhe, Daniel Ortega-Quijano. Transverse leukonychia (Mees' lines) nail alterations in a COVID-19 patient. Dermatol Ther. 2020 Jun 17;e13863

4 : Silvia Méndez-Flores, Angela Zaladonis, Rodrigo Valdes-Rodriguez COVID-19 and nail manifestation: be on the lookout for the red half-moon nail sign. Int J Dermatol2020 Aug 29.

5 : Saud Alobaida, Joseph M Lam Beau lines associated with COVID-19. Case Reports. CMAJ 2020 Sep 8;192(36):E1040.

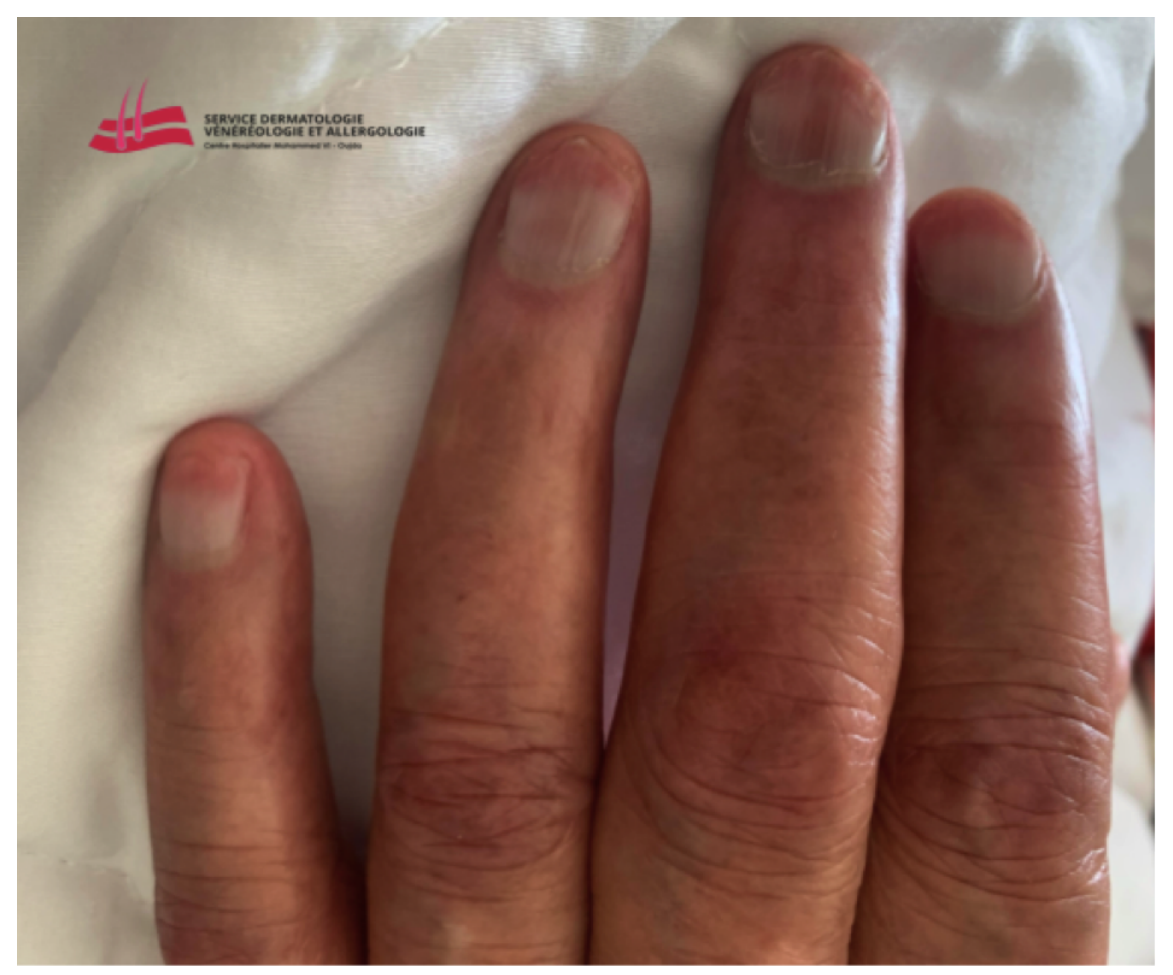




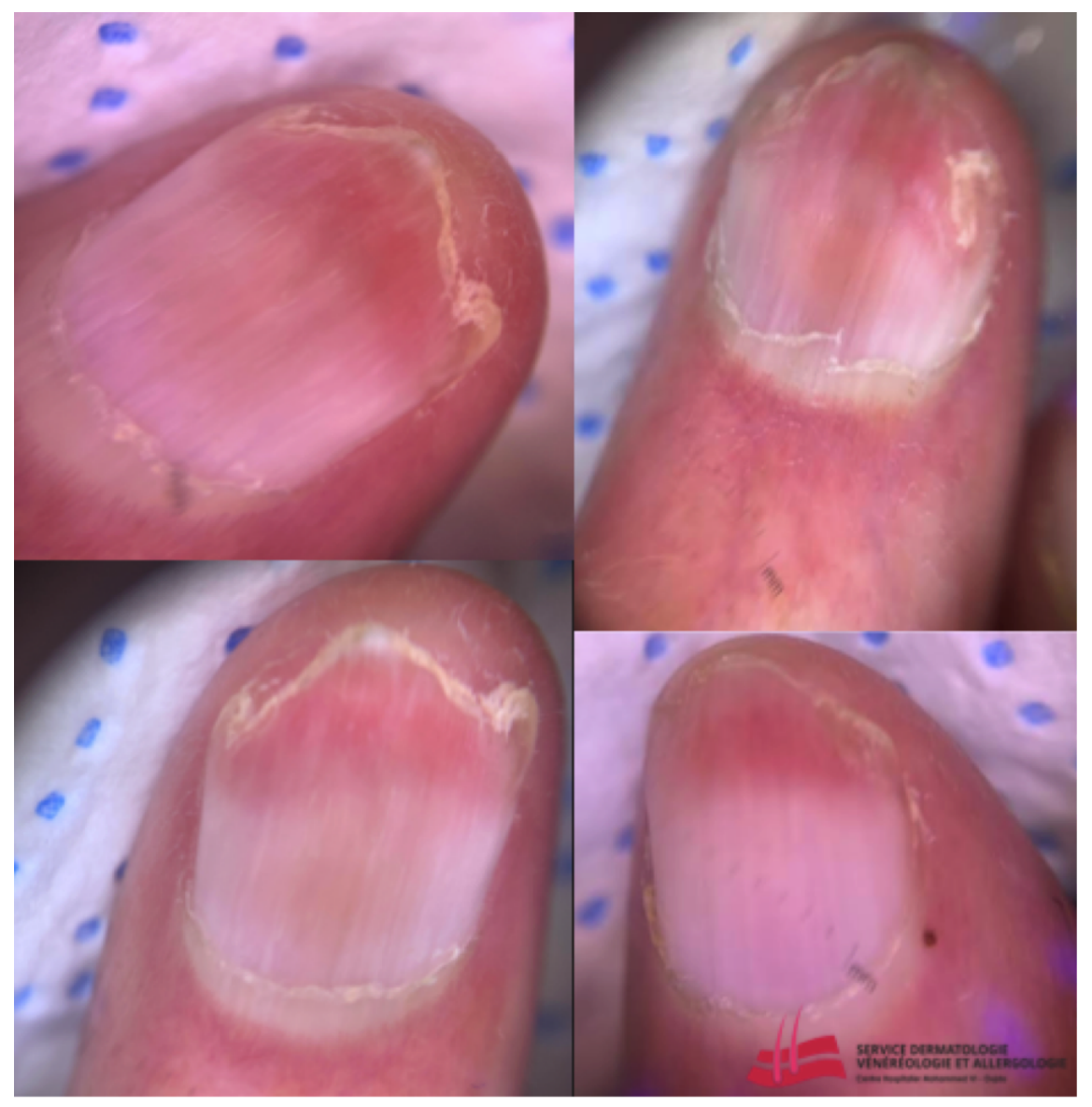

